TAIWANESE JOURNAL OF MATHEMATICS

Vol. 15, No. 1, pp. 307-330, February 2011

This paper is available online at http://www.tjm.nsysu.edu.tw/

\title{
FIXED POINT THEOREMS ON PRODUCT TOPOLOGICAL SEMILATTICE SPACES, GENERALIZED ABSTRACT ECONOMIES AND SYSTEMS OF GENERALIZED VECTOR QUASI-EQUILIBRIUM PROBLEMS
}

\author{
Suliman Al-Homidan and Qamrul Hasan Ansari
}

\begin{abstract}
In this paper, we establish fixed point theorems for a family of multivalued maps defined on the product space of topological semilattice spaces. By using our fixed point theorems, we derive a result on the nonempty intersection of sets without convex structure and equilibrium existence theorems for generalized abstract economies with two constraint correspondences. We present some special cases of our results which generalize several known results in the literature. We consider systems of generalized vector quasi-equilibrium problems and their special cases. As an application of our equilibrium existence theorems, we establish some existence results for solutions of systems of generalized vector quasi-equilibrium problems and their special cases. The results of this paper improve and extend several results in the literature.
\end{abstract}

\section{INTRODUCTION}

Browder type fixed point theorems for a family of multivalued maps play a vital role in proving the existence of solutions of several problems, namely, systems of variational inequalities, systems of equilibrium problems, systems of quasiequilibrium problems, equilibrium existence theorems for abstract economies, Nash equilibrium problem, etc. and establishing nonempty intersection theorems of sets with convex sections. Tarafdar [41] established a fixed point theorem for a family

Received March 1, 2009, accepted June 3, 2009.

Communicated by J. C. Yao.

2000 Mathematics Subject Classification: Primary 47H10, 90C29, 49J40, 91A10; Secondary 47J20, 91B52, 54C60, 54H25.

Key words and phrases: Fixed point theorems, Generalized abstract economies, Intersection theorems, Systems of generalized vector quasi-equilibrium problems, Topological semilattice spaces.

This research was done during the stay of second author at KFUPM and supported by the Fast Track Research Project No. \# FT070007 of King Fahd University of Petroleum \& Minerals, Dhahran, Saudi Arabia. Authors are grateful to the Department of Mathematics and Statistics, King Fahd University of Petroleum \& Minerals, Dhahran, Saudi Arabia for providing excellent research facilities to carry out this work. 
of multivalued maps defined on the product space of compact convex subsets of topological vector spaces and gave some applications to mathematical economies, game theory and problems of social sciences. Ansari and Yao [7], Lan and Webb [25], Lin et al. [28, 32] and Singh et al. [39] extended and generalized the result of Tarafdar [41] for noncompact settings. Ding et al. [15, 17] established some collectively fixed point theorems in the setting of general topological spaces.

In 2004, Ansari et al. [3] introduced the concept of system of vector quasiequilibrium problems (in short, (SVQEP)) which is the extension of system of vector equilibrium problems [5]. They studied the existence of solutions of (SVQEP) and gave some applications to the system of vector quasi-variational inequalities, system of vector quasi-optimization problems, vector quasi-saddle point problem and constrained Nash equilibrium problem for vector-valued functions with infinite number of players. Recently, system of generalized vector quasi-equilibrium problems (in short, (SGVQEP)) is introduced and studied in $[4,15,20]$ because of its application to constrained Nash equilibrium problem for nondifferentiable (in some sense) and nonconvex vector-valued functions. Later, it is also considered and studied by Ding and Yao [19] and Lin and Yu [33] in the setting of $G$-convex spaces. Very recently, Lin [27] studied (SGVQEP) and gave some of its applications to fixed point theorems for a family of nonexpensive multivalued maps, mathematical programs with equilibrium constraints, semi-infinite programs and bilevel programming problems. Very recently, (SGVQEP) for three variables multivalued maps are considered and studied by Lin et al. [29] and Peng et al. [36]. They studied the existence of solutions of such problems and gave an application to Debreu type equilibrium problem for vector-valued functions.

In this paper, we establish fixed point theorems for a family of multivalued maps defined on the product space of topological semilattice spaces. These results extend and generalize several results in the literature. By using our fixed point theorems, we derive a result on the nonempty intersection of sets and equilibrium existence theorems for generalized abstract economies with two constraint correspondences. We present some special cases of our results which generalize several known results in the literature. We consider systems of generalized vector quasi-equilibrium problems for three variables multivalued maps and we also consider their special cases. As an application of our equilibrium existence theorems, we establish some existence results for solutions of our systems of generalized vector quasi-equilibrium problems and their special cases. The results of this paper improve, generalize and extend several results in the literature.

\section{PRELIMINARIES}

Throughout the paper, unless otherwise specified, we denote by 


$$
\Delta_{\ell}=\left\{\left(a_{0}, \ldots, a_{\ell}\right) \in \mathbb{R}_{+}^{\ell+1}: \sum_{i=0}^{\ell} a_{i}=1\right\}
$$

the $\ell$-dimensional simplex. For $J \subseteq\{0,1, \ldots, \ell\}$, we denote by $\Delta_{J}$ the face of $\Delta_{\ell}$ spanned by the unit vectors $e_{j}$ for $j \in J$, that is, if $J=\left\{i_{0}, \ldots, i_{k}\right\} \subseteq\{0,1, \ldots, \ell\}$, then $\Delta_{J}=\left\{t \in \Delta_{\ell}: \sum_{j=0}^{k} t_{i_{j}}=1\right\}$. The family of all subsets (respectively, all nonempty finite subsets) of a set $D$ is denoted by $2^{D}$ (respectively, $\langle D\rangle$ ). If $D$ is a subset of a topological space, then $\bar{D}$ and int $D$ denote the closure and interior of $D$, respectively.

A semilattice is a partially ordered set $X$, with the partial ordering denoted by $\leq$, for which every pair $\left(x, x^{\prime}\right)$ of elements has a least upper bound, denoted by $x \vee x^{\prime}$. It is easy to see that any nonempty finite subset $N$ of $X$ has a least upper bound, denoted by $\sup N$. In a partially ordered set $(X, \leq)$, two arbitrary elements $x$ and $x^{\prime}$ do not have to be comparable. In the case $x \leq x^{\prime}$, the set $\left[x, x^{\prime}\right]=\left\{y \in X: x \leq y \leq x^{\prime}\right\}$ is called an ordered interval. We assume that $(X, \leq)$ is a semilattice and $N$ is a nonempty finite subset of $X$, then the set $\Delta(N)=\bigcup_{n \in N}[n, \sup N]$ is well defined and it has the following properties:

(a) $N \subseteq \Delta(N)$;

(b) If $N \subseteq M$, then $\Delta(N) \subseteq \Delta(M)$.

A subset $E$ of $X$ is called $\Delta$-convex [23] if for any nonempty finite subset $N \subseteq E$, we have $\Delta(N) \subseteq E$.

Let $\mathrm{C}$ be the family of $\Delta$-convex subsets of a semilattice $X$. If $N$ is an arbitrary subset of $X$, then $\Delta$-convex hull of $N$ is defined as $\mathrm{CO}_{\Delta}(N)=\bigcap\{E \in$ C : $N \subseteq E\}$.

It is easy to see that

(a) $N \subseteq \mathrm{CO}_{\Delta}(N)$,

(b) if $N \subseteq M$, then $\mathrm{CO}_{\Delta}(N) \subseteq \mathrm{CO}_{\Delta}(M)$,

(c) $\mathrm{CO}_{\Delta}\left(\mathrm{CO}_{\Delta}(N)\right)=\mathrm{CO}_{\Delta}(N)$,

(d) $\mathrm{CO}_{\Delta}(M \cap N) \subseteq \mathrm{CO}_{\Delta}(M) \cap \mathrm{CO}_{\Delta}(N)$,

(e) arbitrary product of $\Delta$-convex sets is $\Delta$-convex.

Furthermore, a subset $N$ of $X$ is $\Delta$-convex if and only if $\mathrm{CO}_{\Delta}(N)=N$.

One can also see without difficulty that $N$ is $\Delta$-convex if and only if the following conditions are satisfied:

(a) If $x, x^{\prime} \in N$, then $x \vee x^{\prime} \in N$; 
(b) If $x, x^{\prime} \in N$ and $x \leq x^{\prime}$, then $\left[x, x^{\prime}\right] \subseteq N$.

It can be easily verified that the arbitrary intersection of $\Delta$-convex sets is $\Delta$-convex. Therefore, $\mathrm{CO}_{\Delta}(N)$ is the smallest $\Delta$-convex set containing $N$. We can also easily prove that for any finite subset $M$ of $N, \Delta(M) \subseteq \mathrm{CO}_{\Delta}(N)$.

A topological semilattice is a topological space $X$ with a partial ordering $\leq$ for which it is a semilattice with a continuous sup operation (that is, the function $X \times X \rightarrow X,\left(x, x^{\prime}\right) \mapsto x \vee x^{\prime}$ is continuous).

The following result is contained in the proof of Theorem 1 in [23].

Lemma 2.1. Let $X$ be a topological semilattice with path-connected intervals. Then, for any $J \subseteq\{0,1, \ldots, \ell\}$ and any $\left\{x_{j}: j \in J\right\} \subseteq X$, there exists a continuous function $f: \Delta_{\ell} \rightarrow X$ such that $f\left(\Delta_{J}\right) \subseteq \Delta\left(\left\{x_{j}: j \in J\right\}\right)$.

A nonempty subset $D$ of a topological space $X$ is said to be compactly open (respectively, compactly closed) if for every nonempty compact subset $C$ of $X$, $D \cap C$ is open (respectively, closed) in $C$. The compact closure and compact interior of $D$ [12], denoted by ccl $D$ and cint $D$, are defined as

ccl $D=\cap\{G: D \subseteq G$ and $G$ is compactly closed in $X\}$, cint $D=\cup\{G: G \subseteq D$ and $G$ is compactly open in $X\}$.

It is easy to see that cint $D$ is a compactly open set in $X$ and for each nonempty compact subset $C$ of $X$ with $D \cap C \neq \emptyset$, we have $(\operatorname{cint} D) \cap C=\operatorname{int}_{C}(D \cap C)$, where $\operatorname{int}_{C}(D \cap C)$ denotes the interior of $D \cap C$ in $C$. It is clear that a subset $D$ of $X$ is compactly open (respectively, compactly closed in $X$ ) if and only if cint $D=D$ (respectively, ccl $D=D$ ).

Definition 2.1. Let $X$ and $Y$ be topological vector spaces. A multivalued map $T: X \rightarrow 2^{Y} \backslash\{\emptyset\}$ is said to be

(a) closed if its graph is closed in $X \times Y$;

(b) upper semicontinuous on $X$ if for each $x_{0} \in X$ and for any open set $V$ in $Y$ containing $T\left(x_{0}\right)$ there exists an open neighborhood $U$ of $x_{0}$ in $X$ such that $T(x) \subseteq V$ for all $x \in U$;

(c) lower semicontinuous on $X$ if for all $x \in X, y \in T(x)$ and for any $x_{n} \in X$ such that $x_{n} \rightarrow x$, there exists $y_{n} \in T\left(x_{n}\right)$ such that $y_{n} \rightarrow y$.

It is well known that if $X$ is compact and $T: X \rightarrow 2^{Y} \backslash\{\emptyset\}$ is upper semicontinuous multivalued map with compact values, then $T(X)$ is compact.

Let $X$ and $Y$ be topological spaces. A multivalued map $T: X \rightarrow 2^{Y}$ is said to be transfer compactly open valued on $X$ [14] if for every $x \in X$ and for any 
compact subset $D$ of $Y$ with $T(x) \cap D \neq \emptyset, y \in T(x) \cap D$ implies that there exists a point $\hat{x} \in X$ such that $y \in \operatorname{int}_{D}(T(\hat{x}) \cap D)$. T is said to be transfer open valued on $X$ if for every $x \in X, y \in T(x)$, there exists a point $\hat{x} \in X$ such that $y \in \operatorname{int} T(\hat{x})$.

$T$ is said to have the compactly local intersection property [13] if for each nonempty compact subset $D$ of $X$ and for each $x \in D$ with $T(x) \neq \emptyset$, there exists an open neighborhood $N(x)$ of $x$ such that $\bigcap_{z \in N(x) \cap D} T(z) \neq \emptyset$.

By using the argument of Lemma 2.1 in [10], we can easily establish the following result.

Lemma 2.2. Let $X$ and $Y$ be two topological spaces and let $G: X \rightarrow 2^{Y}$ be a multivalued map. Then $G$ is transfer compactly open valued if and only if

$$
\bigcup_{x \in X} G(x)=\bigcup_{x \in X} \operatorname{cint} G(x) .
$$

By applying Lemma 2.2 and following the argument of Proposition 1 [31] and Lemma 1.1 in [13], we have the following lemma.

Lemma 2.3. Let $X$ and $Y$ be two topological spaces and let $G: X \rightarrow 2^{Y}$ be a multivalued map. Then the following statements are equivalent:

(i) $G$ has the compactly local intersection property.

(ii) $G^{-1}: Y \rightarrow 2^{X}$ is transfer compactly open valued and for all $x \in X, G(x)$ is nonempty.

(iii) $X=\bigcup_{y \in Y} \operatorname{cint} G^{-1}(y)$.

\section{Fixed Point Theorems on Product of Topological Semilattice Spaces}

Rest of the paper, unless otherwise specified, for each $i \in I$, we assume that $X_{i}$ is a topological semilattice space with path-connected intervals. We set $X=\prod_{i \in I} X_{i}$, $X^{i}=\prod_{j \in I, j \neq i} X_{j}$ and we write $X=X^{i} \otimes X_{i}$. For each fixed $i \in I$ and $x \in X$, we also write $x=\left(x^{i}, x_{i}\right)=\left(x_{i}\right)_{i \in I}$, where $x^{i}$ and $x_{i}$ denote the projection of $x$ onto $X^{i}$ and $X_{i}$, respectively.

We present a Browder type fixed point theorem for a family of multivalued maps defined on product spaces of noncompact topological semilattice spaces.

Theorem 3.1. For each $i \in I$, let $S_{i}, T_{i}: X^{i} \rightarrow 2^{X_{i}}$ be multivalued maps such that the following conditions are satisfied: 
(i) For all $x^{i} \in X^{i}, S_{i}\left(x^{i}\right) \neq \emptyset, S_{i}\left(x^{i}\right) \subseteq T_{i}\left(x^{i}\right)$ and if $y_{i}, z_{i} \in S_{i}\left(x^{i}\right)$ then $\left[y_{i}, y_{i} \vee z_{i}\right] \subseteq S_{i}\left(x^{i}\right)$.

(ii) For each compact subset $D^{i} \subseteq X^{i}, D^{i}=\bigcup_{y_{i} \in X_{i}}\left(\operatorname{cint} S_{i}^{-1}\left(y_{i}\right) \cap D^{i}\right)$, where $S_{i}^{-1}\left(y_{i}\right)=\left\{x^{i} \in X^{i}: y_{i} \in S_{i}\left(x^{i}\right)\right\}$.

(iii) There exists a nonempty subset $X_{i}^{0} \subseteq X_{i}$ such that for all $M_{i} \in\left\langle X_{i}\right\rangle$, there exists a compact $\Delta$-convex subset $L_{M_{i}} \subseteq X_{i}$ containing $X_{i}^{0} \cup M_{i}$ such that the set $K^{i}=\bigcap_{y_{i} \in X_{i}^{0}}\left(\operatorname{cint} S_{i}^{-1}\left(y_{i}\right)\right)^{c}$ is empty or compact in $X^{i}$, where $\left(\operatorname{cint} S_{i}^{-1}\left(y_{i}\right)\right)^{c}$ denotes the complement of $\operatorname{cint} S_{i}^{-1}\left(y_{i}\right)$ in $X^{i}$.

Then there exists $\bar{x}=\left(\bar{x}^{i}, \bar{x}_{i}\right) \in X$ such that $\bar{x}_{i} \in T_{i}\left(\bar{x}^{i}\right)$ for each $i \in I$.

Proof. For each $i \in I$, if $K^{i}=\emptyset$, then clearly

$$
X^{i}=\bigcup_{y_{i} \in X_{i}^{0}} \operatorname{cint} S_{i}^{-1}\left(y_{i}\right) .
$$

For each $i \in I$, if $K^{i}$ is nonempty and compact, then by condition (ii)

$$
K^{i}=\bigcap_{y_{i} \in X_{i}^{0}}\left(\operatorname{cint} S_{i}^{-1}\left(y_{i}\right)\right)^{c} \subseteq \bigcup_{y_{i} \in X_{i}} \operatorname{cint} S_{i}^{-1}\left(y_{i}\right) .
$$

Since $K^{i}$ is compact, for each $i \in I$, there exists a finite set $M_{i}=\left\{y_{i_{0}}, y_{i_{1}}, \ldots, y_{i_{m_{i}}}\right\} \in$ $\left\langle X_{i}\right\rangle$ such that

$$
K^{i}=\bigcap_{y_{i} \in X_{i}^{0}}\left(\operatorname{cint} S_{i}^{-1}\left(y_{i}\right)\right)^{c} \subseteq \bigcup_{k=0}^{m_{i}} \operatorname{cint} S_{i}^{-1}\left(y_{i_{k}}\right) .
$$

Since $X^{i} \backslash K^{i}=\bigcup_{y_{i} \in X_{i}^{0}} \operatorname{cint} S_{i}^{-1}\left(y_{i}\right)$, we have

$$
X^{i}=\left(\bigcup_{y_{i} \in X_{i}^{0}} \operatorname{cint} S_{i}^{-1}\left(y_{i}\right)\right) \cup\left(\bigcup_{k=0}^{m_{i}} \operatorname{cint} S_{i}^{-1}\left(y_{i_{k}}\right)\right) .
$$

Therefore, in both the cases, either $K^{i}$ is empty or nonempty and compact, (3.1) holds.

By condition (iii), for the finite set $M_{i}$, there exists a compact $\Delta$-convex subset $L_{M_{i}}$ of $X_{i}$ containing $X_{i}^{0} \cup M_{i}$. From (3.1), we have

$$
X^{i}=\bigcup_{y_{i} \in L_{M_{i}}} \operatorname{cint} S_{i}^{-1}\left(y_{i}\right) .
$$


Let $M=\prod_{i \in I} M_{i}, L_{M}=\prod_{i \in I} L_{M_{i}}$ and $L_{M}^{i}=\prod_{j \in I \quad j \neq i} L_{M_{j}}$ for each $i \in I$. Then, for each $i \in I, L_{M}^{i}$ is a compact and $\Delta$-convex subset of $X^{i}$. By (3.2), we have for each $i \in I$,

$$
L_{M}^{i} \subseteq \bigcup_{y_{i} \in L_{M_{i}}} \operatorname{cint} S_{i}^{-1}\left(y_{i}\right) .
$$

Since $L_{M}^{i}$ is compact, for each $i \in I$, there exists a finite set $N_{i}=\left\{y_{i_{0}}, \ldots, y_{i_{m_{i}}}\right\}$ of $L_{M_{i}}$ such that

$$
L_{M}^{i} \subseteq \bigcup_{k=0}^{m_{i}} \operatorname{cint} S_{i}^{-1}\left(y_{i_{k}}\right) .
$$

Since $L_{M}^{i}$ is compact, there exists a continuous partition of unity $\left\{\beta_{i_{0}}, \ldots, \beta_{i_{m_{i}}}\right\}$ subordinated to the open covering $\left\{\operatorname{cint} S_{i}^{-1}\left(y_{i_{k}}\right)\right\}_{k=0}^{m_{i}}$, that is, for each $k=$ $0,1 \ldots, m_{i}, \beta_{i_{k}}: L_{M}^{i} \rightarrow[0,1]$ is continuous such that for all $x^{i} \in L_{M}^{i}, \sum_{k=0}^{m_{i}} \beta_{i_{k}}\left(x^{i}\right)=$ 1 and for each $k=0,1, \ldots, m_{i}, \beta_{i_{k}}\left(x^{i}\right)=0$ for $x^{i} \notin \operatorname{cint} S_{i}^{-1}\left(y_{i_{k}}\right)$. In other words, $\beta_{i_{k}}\left(x^{i}\right) \neq 0$ implies $x^{i} \in \operatorname{cint} S_{i}^{-1}\left(y_{i_{k}}\right) \subseteq S_{i}^{-1}\left(y_{i_{k}}\right)$, that is, $y_{i_{k}} \in S_{i}\left(x^{i}\right)$ for all $k=0,1, \ldots, m_{i}$ and for each $i \in I$. For each $i \in I$, let $\varphi_{i}: L_{M}^{i} \rightarrow \Delta_{m_{i}}$ be a map defined as

$$
\varphi_{i}\left(x^{i}\right)=\sum_{k=0}^{m_{i}} \beta_{i_{k}}\left(x^{i}\right) e_{i_{k}} \quad \text { for all } x^{i} \in L_{M}^{i},
$$

where $\left\{e_{i_{k}}: k=0,1, \ldots, m_{i}\right\}$ is the set of vertices of the standard $m_{i}$-dimensional simplex $\Delta_{m_{i}}$. Then clearly for each $i \in I, \varphi_{i}$ is continuous.

By Lemma 2.1, for each $i \in I$, there exists a continuous function $g_{i}: \Delta_{m_{i}} \rightarrow X^{i}$ such that $g_{i}\left(\Delta_{J}\right) \subseteq \Delta\left(\left\{y_{i_{k}}: k \in J\right\}\right)$ for any nonempty subset $J \subseteq\left\{0,1, \ldots, m_{i}\right\}$.

For all $x^{i} \in L_{M}^{i}$, let $P_{i}\left(x^{i}\right)=\left\{j \in\left\{0,1, \ldots, m_{i}\right\}: \beta_{i_{j}}\left(x^{i}\right) \neq 0\right\}$. Then for all $x^{i} \in L_{M}^{i}, \varphi_{i}\left(x^{i}\right)=\sum_{k \in P_{i}\left(x^{i}\right)} \beta_{i_{k}}\left(x^{i}\right) e_{i_{k}} \in \Delta_{P_{i}\left(x^{i}\right)}$. From the last part of condition (i), for all $x^{i} \in L_{M}^{i}, \Delta\left(\left\{y_{i_{k}}: k \in P_{i}\left(x^{i}\right)\right\}\right) \subseteq S_{i}\left(x^{i}\right)$. Therefore, for all $x^{i} \in L_{M}^{i}$,

$$
\left(g_{i} \circ \varphi_{i}\right)\left(x^{i}\right) \in g_{i}\left(\Delta_{P_{i}\left(x^{i}\right)}\right) \subseteq \Delta\left(\left\{y_{i_{k}}: k \in P_{i}\left(x^{i}\right)\right\}\right) \subseteq S_{i}\left(x^{i}\right) \subseteq T_{i}\left(x^{i}\right) .
$$

For each $i \in I$, let $E_{i}$ be the linear hull of $\left\{e_{i_{k}}: k=0,1, \ldots, m_{i}\right\}$, then $E_{i}$ is a locally convex topological vector space as it is finite dimensional and $\Delta_{m_{i}}$ is a compact convex subset of $E_{i}$. Let $E=\prod_{i \in I} E_{i}$, then $E$ is also a locally convex topological vector space and $C=\prod_{i \in I} \Delta_{m_{i}}$ is a compact convex subset of $E$. Define two mappings $h: C \rightarrow L_{M}$ and $\Psi: L_{M} \rightarrow C$ by

$$
h(z)=\prod_{i \in I} g_{i}\left(\pi_{i}(z)\right) \quad \text { for all } z \in C
$$


and

$$
\Psi(x)=\prod_{i \in I} \varphi_{i}\left(x^{i}\right) \quad \text { for all } x=\left(x^{i}, x_{i}\right) \in L_{M},
$$

where $\pi_{i}: C \rightarrow \Delta_{m_{i}}$ is the projection of $C$ onto $\Delta_{m_{i}}$. Since for each $i \in I, g_{i}$ and $\varphi_{i}$ are continuous, $h$ and $\Psi$ are also continuous. Therefore, $\Psi \circ h=F: C \rightarrow C$ is a well-defined and continuous function. By Tychonoff's fixed point theorem [42], there exists $\bar{u} \in C$ such that $\bar{u}=F(\bar{u})=(\Psi \circ h)(\bar{u})$. Let $\bar{x}=\left(\bar{x}_{i}\right)_{i \in I}=h(\bar{u})$, then $\bar{u}=\Psi(\bar{x})$ and

$\bar{x}=h(\Psi(\bar{x}))=h\left(\prod_{i \in I} \varphi_{i}\left(\bar{x}^{i}\right)\right)=\prod_{i \in I} g_{i}\left(\pi_{i}\left(\prod_{i \in I} \varphi_{i}\left(\bar{x}^{i}\right)\right)\right)=\prod_{i \in I}\left(g_{i} \circ \varphi_{i}\right)\left(\bar{x}^{i}\right)$.

Therefore, $\bar{x}_{i}=\left(g_{i} \circ \varphi_{i}\right)\left(\bar{x}^{i}\right) \in T_{i}\left(\bar{x}^{i}\right)$ for each $i \in I$.

If for each $i \in I, X^{i}$ is replaced by $X$ in Theorem 3.1, then by using the same argument as in the proof of Theorem 3.1, the following result can be easily established.

Theorem 3.2. For each $i \in I$, let $S_{i}, T_{i}: X \rightarrow 2^{X_{i}}$ be multivalued maps such that the following conditions are satisfied:

(i) For all $x \in X, S_{i}(x) \neq \emptyset, S_{i}(x) \subseteq T_{i}(x)$ and if $y_{i}, z_{i} \in S_{i}(x)$ then $\left[y_{i}, y_{i} \vee z_{i}\right] \subseteq S_{i}(x)$.

(ii) For each compact subset $D \subseteq X, D=\bigcup_{y_{i} \in X_{i}}\left(\operatorname{cint} S_{i}^{-1}\left(y_{i}\right) \cap D\right)$.

(iii) There exists a nonempty subset $X_{i}^{0} \subseteq X_{i}$ such that for all $M_{i} \in\left\langle X_{i}\right\rangle$, there exists a compact $\Delta$-convex subset $L_{M_{i}} \subseteq X_{i}$ containing $X_{i}^{0} \cup M_{i}$ such that the set $K=\bigcap_{y_{i} \in X_{i}^{0}}\left(\operatorname{cint} S_{i}^{-1}\left(y_{i}\right)\right)^{c}$ is empty or compact in $X$, where $\left(\operatorname{cint} S_{i}^{-1}\left(y_{i}\right)\right)^{c}$ denotes the complement of $\operatorname{cint} S_{i}^{-1}\left(y_{i}\right)$ in $X$.

Then there exists $\bar{x} \in X$ such that $\bar{x}_{i} \in T_{i}(\bar{x})$ for each $i \in I$.

Remark 3.1. In view of Lemmas 2.2 and 2.3, condition (ii) in Theorems 3.1 and 3.2 can be replaced by any one of the following conditions:

(ii)' For each $i \in I, S_{i}^{-1}$ is transfer compactly open valued on $K$.

(ii)" For each $i \in I$ and for all $y_{i} \in K_{i}, S_{i}^{-1}\left(y_{i}\right)$ is compactly open in $K$.

(ii) $)^{\prime \prime \prime}$ For each $i \in I, S_{i}$ has the compactly local intersection property.

When $I$ is a singleton set, we obtain the following fixed point result as a consequence of Theorem 3.1. 
Corollary 3.1. Let $X$ be a topological semilattice space with path-connected intervals. Let $S, T: X \rightarrow 2^{X}$ be multivalued maps such that the following conditions are satisfied:

(i) For all $x \in X, S(x) \neq \emptyset, S(x) \subseteq T(x)$ and if $y, z \in S(x)$ then $[y, y \vee z] \subseteq$ $S(x)$.

(ii) For each compact subset $D \subseteq X, D=\bigcup_{y \in X}\left(\operatorname{cint} S^{-1}(y) \cap D\right)$.

(iii) There exists a nonempty subset $X^{0} \subseteq X$ such that for all $M \in\langle X\rangle$, there exists a compact $\Delta$-convex subset $L_{M} \subseteq X$ containing $X^{0} \cup M$ such that the set $K=\bigcap_{y \in X^{0}}\left(\operatorname{cint} S^{-1}(y)\right)^{c}$ is empty or compact in $X$, where $\left(\operatorname{cint} S^{-1}(y)\right)^{c}$ denotes the complement of $\operatorname{cint} S^{-1}(y)$ in $X$.

Then there exists $\bar{x} \in K$ such that $\bar{x} \in T(\bar{x})$.

Remark 3.2. In view of Remark 3.1, Corollary 3.1 generalizes Corollary 1 in [23] and Theorem 2.4 in [34] to noncompact setting. Corollary 3.1 extends Corollary 1 in [7], Theorem 2 in [16], Theorem 1 in [40], Corollary 3 in [43], Theorem 3.2 in [44] and several other results in the literature to topological semilattice space setting.

By using the same argument as in the proof of Theorem 3.1, we can easily establish the following result.

Theorem 3.3. For each $i \in I$, let $X_{i}$ be a topological semilattice space with path-connected intervals, $K_{i}$ be a nonempty $\Delta$-convex subset of $X_{i}$ and $K=$ $\prod_{i \in I} K_{i}$. For each $i \in I$, let $S_{i}, T_{i}: K \rightarrow 2^{K_{i}}$ be multivalued maps such that the following conditions are satisfied:

(i) For all $x \in K, S_{i}(x) \neq \emptyset, \mathrm{CO}_{\Delta}\left(S_{i}(x)\right) \subseteq T_{i}(x)$.

(ii) For each compact subset $D \subseteq K, D=\bigcup_{y_{i} \in K_{i}}\left(\operatorname{cint} S_{i}^{-1}\left(y_{i}\right) \cap D\right)$.

(iii) There exists a nonempty subset $K_{i}^{0} \subseteq K_{i}$ such that for all $M_{i} \in\left\langle K_{i}\right\rangle$, there exists a compact $\Delta$-convex subset $L_{M_{i}} \subseteq K_{i}$ containing $K_{i}^{0} \cup M_{i}$ such that the set $\mathcal{K}=\bigcap_{y_{i} \in K_{i}^{0}}\left(\operatorname{cint} S_{i}^{-1}\left(y_{i}\right)\right)^{c}$ is empty or compact in $K$.

Then there exists $\bar{x} \in K$ such that $\bar{x}_{i} \in T_{i}(\bar{x})$ for each $i \in I$.

Remark 3.3. The condition (iii) in Theorem 3.3 can be replaced by the following condition. 


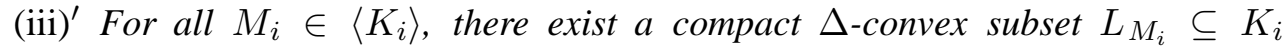
containing $M_{i}$ and a nonempty compact set $\mathcal{D}$ of $K$ such that for each $x \in K \backslash \mathcal{D}$, there exists a $\tilde{y}_{i} \in L_{M_{i}}$ satisfying $x \in \operatorname{cint} S_{i}^{-1}\left(\tilde{y}_{i}\right)$.

Proof. For each $i \in I$, let $K_{i}^{0}=L_{M_{i}} \subseteq K_{i}$. Then from condition (iii)', we have

$$
\bigcap_{y_{i} \in K_{i}^{0}}\left(\operatorname{cint} S_{i}^{-1}\left(y_{i}\right)\right)^{c}=K \backslash \bigcup_{y_{i} \in L_{M_{i}}} \operatorname{cint} S_{i}^{-1}\left(y_{i}\right) \subseteq \mathcal{D} \text {. }
$$

If $\bigcap_{y_{i} \in K_{i}^{0}}\left(\operatorname{cint} S_{i}^{-1}\left(y_{i}\right)\right)^{c} \neq \emptyset$, then it is a compactly closed subset of the compact set $\mathcal{D}$ and hence it is compact. Thus, condition (iii) of Theorem 3.3 holds.

Remark 3.4. In view of Remarks 3.1 and 3.3, Theorem 3.3 extends and generalizes Theorem 1 in [7], Theorem 2.4 in [17], Theorem 2.1 in [25], Theorem 3.1 in [32], Theorem 2.1 [39] and several other results in the literature.

\section{A Result on Nonempty Intersection of Sets and \\ Generalized Abstract ECONOMIES}

We establish the following nonempty intersection theorem of sets with or without $\Delta$-convex sections by using Theorem 3.1.

Theorem 4.1. Let $\left\{A_{i}\right\}_{i \in I}$ and $\left\{B_{i}\right\}_{i \in I}$ be two families of subsets of $X$ such that for each $i \in I$, the following conditions hold:

(i) For all $x^{i} \in X^{i},\left\{y_{i} \in X_{i}:\left(x^{i}, y_{i}\right) \in A_{i}\right\} \subseteq\left\{y_{i} \in X_{i}:\left(x^{i}, y_{i}\right) \in B_{i}\right\}$ and if $y_{i}, z_{i} \in\left\{y_{i} \in X_{i}:\left(x^{i}, y_{i}\right) \in A_{i}\right\}$ then $\left[y_{i}, y_{i} \vee z_{i}\right] \subseteq\left\{y_{i} \in X_{i}:\left(x^{i}, y_{i}\right) \in\right.$ $\left.A_{i}\right\}$.

(ii) For each compact subset $D^{i} \subseteq X^{i}$,

$$
D^{i}=\bigcup_{y_{i} \in X_{i}}\left(\operatorname{cint}\left\{x^{i} \in X^{i}:\left(x^{i}, y_{i}\right) \in A_{i}\right\} \cap D^{i}\right) .
$$

(iii) There exists a nonempty subset $X_{i}^{0} \subseteq X_{i}$ such that for all $M_{i} \in\left\langle X_{i}\right\rangle$, there exists a compact $\Delta$-convex subset $L_{M_{i}} \subseteq X_{i}$ containing $X_{i}^{0} \cup M_{i}$ such that the set $\bigcap_{y_{i} \in X_{i}^{0}} \operatorname{ccl}\left\{x^{i} \in X^{i}:\left(x^{i}, y_{i}\right) \notin A_{i}\right\}$ is empty or compact in $X^{i}$.

Then $\bigcap_{i \in I} B_{i} \neq \emptyset$.

Proof. For each $i \in I$ and for all $x^{i} \in X^{i}$, define two multivalued maps $S_{i}, T_{i}: X^{i} \rightarrow 2^{X_{i}}$ as

$$
S_{i}\left(x^{i}\right)=\left\{y_{i} \in X_{i}:\left(x^{i}, y_{i}\right) \in A_{i}\right\} \quad \text { and } \quad T_{i}\left(x^{i}\right)=\left\{y_{i} \in X_{i}:\left(x^{i}, y_{i}\right) \in B_{i}\right\} .
$$


Under the hypothesis, it is easy to verify that all the conditions of Theorem 3.1 are satisfied. Hence by Theorem 3.1, there exists $\bar{x}=\left(\bar{x}^{i}, \bar{x}_{i}\right) \in X$ such that $\bar{x}_{i} \in T_{i}\left(\bar{x}^{i}\right)$ for each $i \in I$, that is, $\bar{x}=\left(\bar{x}^{i}, \bar{x}_{i}\right) \in B_{i}$ for each $i \in I$ and thus $\bigcap_{i \in I} B_{i} \neq \emptyset$.

\section{Remark 4.1.}

(a) Condition (i) in Theorem 4.1 can be replaced by the following condition:

(i) $)^{\prime}$ For all $x^{i} \in X^{i}, \mathrm{CO}_{\Delta}\left(\left\{y_{i} \in X_{i}:\left(x^{i}, y_{i}\right) \in A_{i}\right\}\right) \subseteq\left\{y_{i} \in X_{i}:\left(x^{i}, y_{i}\right) \in B_{i}\right\}$.

(b) Theorem 4.1 generalizes and extends Theorem 16 in [21] and Theorem 2 in [38] to the product space of noncompact topological semilattice spaces.

We define the generalized abstract economies and establish equilibrium existence results.

Because of the fuzziness of consumers' behaviour or market situations, in a real market, any preference of a real agent would be unstable. Therefore, Kim and Tan [24] and Lin et al. [30, 32] studied generalized abstract economy with fuzzy constraint correspondences.

Let $I$ be any set of agents (countable or uncountable). For each $i \in I$, let $K_{i}$ be a nonempty $\Delta$-convex subset of a topological semilattice space with path-connected intervals. Such $K_{i}$ is the set of actions available to the agent $i$. Let $K=\prod_{i \in I} K_{i}$. A generalized abstract economy (or generalized game) $\Gamma=\left(K_{i}, A_{i}, B_{i}, F_{i}, P_{i}\right)_{i \in I}$ is defined as a family of ordered quadruples $\left(K_{i}, A_{i}, B_{i}, F_{i}, P_{i}\right)$ where $A_{i}, B_{i}$ : $K \rightarrow 2^{K_{i}}$ are constraint correspondences, $F_{i}: K \rightarrow 2^{K_{i}}$ is a fuzzy constraint correspondence such that $F_{i}(x)$ is the unstable state for the agent $i$, and $P_{i}: K \times$ $X \rightarrow 2^{K_{i}}$ is a preference correspondence such that $P_{i}(x)$ is the state preference by the agent $i$ at $x$. An equilibrium for $\Gamma$ is a point $(\bar{x}, \bar{y}) \in K \times K$ such that for each $i \in I, \bar{x}_{i} \in B_{i}(\bar{x}), \bar{y}_{i} \in F_{i}(\bar{x})$ and $P_{i}(\bar{x}, \bar{y}) \cap A_{i}(\bar{x})=\emptyset$.

For each $i \in I$, if $A_{i}(x)=B_{i}(x)$ for all $x \in K$, then above generalized game is considered and studied in $[24,30,32]$ in the setting of topological vector spaces.

If for each $i \in I$ and for all $x \in K, F_{i}(x)=K_{i}$ and the preference correspondence $P_{i}$ satisfies $P_{i}(x, y)=P_{i}\left(x, y^{\prime}\right)$ for all $x, y, y^{\prime} \in K$, our definitions of a generalized abstract economy and an equilibrium coincide with the usual definitions of an abstract economy and an equilibrium due Ding et al. [16, 18]. Furthermore, if for each $i \in I$ and for all $x \in K, A_{i}(x)=B_{i}(x)$, then our definitions of a generalized abstract economy and an equilibrium coincide with the usual definitions of an abstract economy and an equilibrium due to Shafer and Sonnenschein [37].

As an application of Theorem 3.3, we derive the following general equilibrium existence result for the generalized abstract economies with infinitely many commodities, infinitely many agents and with general preference correspondences in the setting of topological semilattice spaces. 
Theorem 4.2. For each $i \in I$, let $A_{i}, B_{i}: K \rightarrow 2^{K_{i}}$ be multivalued maps, $P_{i}$ : $K \times K \rightarrow 2^{K_{i}}$ a preference correspondence and $F_{i}: K \rightarrow 2^{K_{i}}$ a fuzzy constraint correspondence. For each $i \in I$, assume that the following conditions hold:

(i) For all $x \in K, \mathrm{CO}_{\Delta}\left(A_{i}(x)\right) \subseteq B_{i}(x)$ and $F_{i}(x)$ is a nonempty $\Delta$-convex set.

(ii) For all $(x, y) \in K \times K, x_{i} \notin \mathrm{CO}_{\Delta}\left(P_{i}(x, y)\right)$,

(iii) For each compact subsets $\tilde{D}$ and $\hat{D}$ of $K$,

$$
\begin{aligned}
\tilde{D} \times \hat{D}= & \bigcup_{\left(u_{i}, v_{i}\right) \in K_{i} \times K_{i}}\left[\operatorname { c i n t } ( P _ { i } ^ { - 1 } ( u _ { i } ) \cup W _ { i } ) \cap \left(\left(A_{i}^{-1}\left(u_{i}\right) \times K\right)\right.\right. \\
& \left.\left.\cap\left(F_{i}^{-1}\left(v_{i}\right) \times K\right)\right)\right] \cap(\tilde{D} \times \hat{D}), \\
& \mathcal{W}_{i}=\left\{(x, y) \in K \times K: P_{i}(x, y) \cap A_{i}(x)=\emptyset\right\} .
\end{aligned}
$$

(iv) There exist nonempty subsets $K_{i}^{0}, K_{i}^{*} \subseteq K_{i}$ such that for all $M_{i}, N_{i} \in\left\langle K_{i}\right\rangle$, there exist compact $\Delta$-convex subset $L_{M_{i}}, L_{N_{i}} \subseteq K_{i}$ containing $K_{i}^{0} \cup M_{i}$ and $K_{i}^{*} \cup N_{i}$, respectively, such that the set

$$
\bigcap_{\left(u_{i}, v_{i}\right) \in K_{i}^{0} \times K_{i}^{*}}\left[\operatorname{cint}\left\{\left(P_{i}^{-1}\left(u_{i}\right) \cup \mathcal{W}_{i}\right) \cap\left(\left(A_{i}^{-1}\left(u_{i}\right) \times K\right) \cap\left(F_{i}^{-1}\left(v_{i}\right) \times K\right)\right)\right\}\right]^{c}
$$

is nonempty or compact.

Then there exists $(\bar{x}, \bar{y}) \in K \times K$ such that for each $i \in I, \bar{x}_{i} \in B_{i}(\bar{x}), \bar{y}_{i} \in F_{i}(\bar{x})$ and $P_{i}(\bar{x}, \bar{y}) \cap A_{i}(\bar{x})=\emptyset$.

Proof. For each $i \in I$, let $\mathcal{V}_{i}=\left\{(x, y) \in K \times K: P_{i}(x, y) \cap A_{i}(x) \neq \emptyset\right\}$, that is, $\mathcal{V}_{i}=(K \times K) \backslash \mathcal{W}_{i}$. For each $i \in I$, define $S_{i}, T_{i}: K \times K \rightarrow 2^{K_{i} \times K_{i}}$ by

$S_{i}(x, y)=\left\{\begin{array}{lll}{\left[P_{i}(x, y) \cap A_{i}(x)\right] \times F_{i}(x),} & \text { if } \quad(x, y) \in \mathcal{V}_{i} \\ A_{i}(x) \times F_{i}(x), & \text { if } \quad(x, y) \in(K \times K) \backslash \mathcal{V}=\mathcal{W}_{i}\end{array}\right.$

and

$T_{i}(x, y)=\left\{\begin{array}{lll}{\left[\mathrm{CO}_{\Delta} P_{i}(x, y) \cap B_{i}(x)\right] \times F_{i}(x),} & \text { if } \quad(x, y) \in \mathcal{V}_{i} \\ B_{i}(x) \times F_{i}(x), & \text { if } \quad(x, y) \in(K \times K) \backslash \mathcal{V}=\mathcal{W}_{i} .\end{array}\right.$

From condition (i), we have $\mathrm{CO}_{\Delta} S_{i}(x, y) \subseteq T_{i}(x, y)$ for each $i \in I$ and for all $(x, y) \in K \times K$. For each $i \in I$ and for all $\left(u_{i}, v_{i}\right) \in K_{i} \times K_{i}$, we have

$$
S_{i}^{-1}\left(u_{i}, v_{i}\right)=\left[P_{i}^{-1}\left(u_{i}\right) \cap \mathcal{W}_{i}\right] \cap\left[\left(A_{i}^{-1}\left(u_{i}\right) \times K\right) \cap\left(F_{i}^{-1}\left(v_{i}\right) \times K\right)\right] .
$$


From condition (iii), for each nonempty compact subsets $\tilde{D}$ and $\hat{D}$ of $K$, we have

$$
\begin{aligned}
\tilde{D} \times \hat{D}= & \bigcup_{\left(u_{i}, v_{i}\right) \in K_{i} \times K_{i}} \operatorname{cint}\left[( P _ { i } ^ { - 1 } ( u _ { i } ) \cup \mathcal { W } _ { i } ) \cap \left(\left(A_{i}^{-1}\left(u_{i}\right) \times K\right)\right.\right. \\
& \left.\left.\cap\left(F_{i}^{-1}\left(v_{i}\right) \times K\right)\right)\right] \cap(\tilde{D} \times \hat{D}) .
\end{aligned}
$$

It follows from condition (iv) that the set

$$
\bigcap_{\left(u_{i}, v_{i}\right) \in K_{i}^{0} \times K_{i}^{*}}\left[\operatorname{cint}\left\{\left(P_{i}^{-1}\left(u_{i}\right) \cup \mathcal{W}_{i}\right) \cap\left(\left(A_{i}^{-1}\left(u_{i}\right) \times K\right) \cap\left(F_{i}^{-1}\left(v_{i}\right) \times K\right)\right)\right\}\right]^{c}
$$

is nonempty or compact. Then by Theorem 3.3, there exists $(\bar{x}, \bar{y}) \in K \times K$ such that $\left(\bar{x}_{i}, \bar{y}_{i}\right) \in T_{i}(\bar{x}, \bar{y})$ for each $i \in I$. If $(\bar{x}, \bar{y}) \in \mathcal{V}_{i}$ for some $i \in I$, then by the definition of $\mathcal{V}_{i}$, we have

$$
\left(\bar{x}_{i}, \bar{y}_{i}\right) \in\left[\mathrm{CO}_{\Delta} P_{i}(\bar{x}, \bar{y}) \cap B_{i}(\bar{x})\right] \times F_{i}(\bar{x})
$$

and hence $\bar{x}_{i} \in \mathrm{CO}_{\Delta} P_{i}(\bar{x}, \bar{y}) \cap B_{i}(\bar{x})$ and so $\bar{x}_{i} \in \mathrm{CO}_{\Delta} P_{i}(\bar{x}, \bar{y})$ which is a contradiction of condition (ii). So, we must have that $(\bar{x}, \bar{y}) \in \mathcal{W}_{i}$ such that $\bar{x}_{i} \in B_{i}(\bar{x})$ and $\bar{y}_{i} \in F_{i}(\bar{x})$ for each $i \in I$. From the definition of $\mathcal{W}_{i}$, we obtain that for each $i \in I, \bar{x}_{i} \in B_{i}(\bar{x})$ and $\bar{y}_{i} \in F_{i}(\bar{x})$ such that $P_{i}(\bar{x}, \bar{y}) \cap A_{i}(\bar{x})=\emptyset$.

By using Remarks 3.1 and 3.3 and Theorem 3.3, the following result can be easily proved on the lines of the proof of Theorem 4.2.

Theorem 4.3. For each $i \in I$, let $A_{i}, B_{i}: K \rightarrow 2^{K_{i}}$ be multivalued maps, $P_{i}: K \times K \rightarrow 2^{K_{i}}$ a preference correspondence and $F_{i}: K \rightarrow 2^{K_{i}}$ a fuzzy constraint correspondence. For each $i \in I$, assume that the following conditions hold:

(i) For all $x \in K, \mathrm{CO}_{\Delta}\left(A_{i}(x)\right) \subseteq B_{i}(x)$ and $F_{i}(x)$ is a nonempty $\Delta$-convex set.

(ii) For all $(x, y) \in K \times K, x_{i} \notin \mathrm{CO}_{\Delta}\left(P_{i}(x, y)\right)$,

(iii) For all $u_{i} \in K_{i}, A_{i}^{-1}\left(u_{i}\right), P_{i}^{-1}\left(u_{i}\right)$ and $F_{i}^{-1}\left(u_{i}\right)$ are compactly open sets.

(iv) The set $\mathcal{W}_{i}=\left\{(x, y) \in K \times K: P_{i}(x, y) \cap A_{i}(x)=\emptyset\right\}$ is compactly open.

(v) For all $M_{i}, N_{i} \in\left\langle K_{i}\right\rangle$, there exist compact $\Delta$-convex subsets $L_{M_{i}}, L_{N_{i}} \subseteq K_{i}$ containing $M_{i}$ and $N_{i}$, respectively, and nonempty compact sets $\tilde{\mathcal{D}}, \mathcal{\mathcal { D }}$ of $K$ such that for each $(x, y) \in K \times K \backslash \tilde{\mathcal{D}} \times \hat{\mathcal{D}}$ and for each $i \in I$, there exists $\left(u_{i}, v_{i}\right) \in L_{M_{i}} \times L_{N_{i}}$ satisfying $u_{i} \in P_{i}(x, y) \cap A_{i}(x)$ and $v_{i} \in F_{i}(x)$.

Then there exists $(\bar{x}, \bar{y}) \in K \times K$ such that for each $i \in I, \bar{x}_{i} \in B_{i}(\bar{x}), \bar{y}_{i} \in F_{i}(\bar{x})$ and $P_{i}(\bar{x}, \bar{y}) \cap A_{i}(\bar{x})=\emptyset$. 
From Theorem 4.3, we can easily derive the following equilibrium existence result for the abstract economies in the setting of topological semilattice spaces.

Corollary 4.1. For each $i \in I$, let $A_{i}: K \rightarrow 2^{K_{i}}$ be a multivalued map and $P_{i}: K \rightarrow 2^{K_{i}}$ a preference correspondence. For each $i \in I$, assume that the following conditions hold:

(i) For all $x \in K, A_{i}(x)$ is nonempty and $\Delta$-convex.

(ii) For all $y_{i} \in K_{i}, A_{i}^{-1}\left(y_{i}\right)$ and $P_{i}^{-1}\left(y_{i}\right)$ are compactly open sets.

(iii) For all $x \in K, x_{i} \notin \mathrm{CO}_{\Delta}\left(P_{i}(x)\right)$.

(iv) The set $\mathcal{W}_{i}=\left\{x \in K: P_{i}(x) \cap A_{i}(x)=\emptyset\right\}$ is compactly open.

(v) For all $M_{i} \in\left\langle K_{i}\right\rangle$, there exist a compact $\Delta$-convex subset $L_{M_{i}} \subseteq K_{i}$ containing $M_{i}$ and a nonempty compact subset $D$ of $K$ such that for all $x \in$ $K \backslash D$ and for each $i \in I$, there exists $\tilde{y}_{i} \in L_{M_{i}}$ satisfying $\tilde{y}_{i} \in P_{i}(x) \cap A_{i}(x)$.

Then there exists $\bar{x} \in K$ such that for each $i \in I, \bar{x}_{i} \in A_{i}(\bar{x})$ and $P_{i}(\bar{x}) \cap A_{i}(x)=\emptyset$.

\section{Remark 4.2.}

(a) Theorems 4.2 and 4.3 extend and improve Theorem 5.1 in [32] and several other results in the literature to topological semilattice space settings and several other aspects.

(b) Corollary 4.1 extends and generalizes Theorem 2 in [7], Theorem 3.3 in [17], Corollary 5.1 in [32] Theorem 3.1 in [41] and several other results in the literature.

\section{Systems of Generalized Vector Quasi-equilibrium Problems}

For each $i \in I$, let $X_{i}$ be a topological semilattice space with path-connected intervals, $K_{i}$ be a nonempty $\Delta$-convex subset of $X_{i}, Y_{i}$ be a topological vector space and let $K=\prod_{i \in I} K_{i}$. For each $i \in I$, let $A_{i}, B_{i}: K \rightarrow 2^{K_{i}}, F_{i}: K \rightarrow 2^{K_{i}}$ be multivalued maps with nonempty values and $C_{i}: K \times K \rightarrow 2^{Y_{i}}$ be a multivalued map such that for all $(x, y) \in K \times K, C_{i}(x, y)$ is a proper closed convex cone with $\operatorname{int} C_{i}(x, y) \neq \emptyset$. For each $i \in I$, let $\Phi_{i}: K \times K \times K_{i} \rightarrow 2^{Y_{i}}$ be a multivalued map with nonempty values. We consider the following systems of generalized vector quasi-equilibrium problems (in short, (SGVQEPs)).

$$
(\operatorname{SGVQEP})(\mathrm{I})\left\{\begin{array}{l}
\text { Find }(\bar{x}, \bar{y}) \in K \times K \text { such that for each } i \in I, \\
\bar{x}_{i} \in B_{i}(\bar{x}), \bar{y}_{i} \in F_{i}(\bar{x}) \text { and } \\
\Phi_{i}\left(\bar{x}, \bar{y}, u_{i}\right) \subseteq C_{i}(\bar{x}, \bar{y}) \quad \text { for all } u_{i} \in A_{i}(\bar{x})
\end{array}\right.
$$




$$
\begin{gathered}
\text { (SGVQEP)(II) }\left\{\begin{array}{l}
\text { Find }(\bar{x}, \bar{y}) \in K \times K \text { such that for each } i \in I, \\
\bar{x}_{i} \in B_{i}(\bar{x}), \bar{y}_{i} \in F_{i}(\bar{x}) \text { and } \\
\Phi_{i}\left(\bar{x}, \bar{y}, u_{i}\right) \cap C_{i}(\bar{x}, \bar{y}) \neq \emptyset \quad \text { for all } u_{i} \in A_{i}(\bar{x}) .
\end{array}\right. \\
\left(\begin{array}{l}
\text { FGVQEP)(III) }(\bar{x}, \bar{y}) \in K \times K \text { such that for each } i \in I, \\
\bar{x}_{i} \in B_{i}(\bar{x}), \bar{y}_{i} \in F_{i}(\bar{x}) \text { and } \\
\Phi_{i}\left(\bar{x}, \bar{y}, u_{i}\right) \cap\left(-\operatorname{int} C_{i}(\bar{x}, \bar{y})\right)=\emptyset \quad \text { for all } u_{i} \in A_{i}(\bar{x}) .
\end{array}\right. \\
\left(\operatorname { S G V Q E P ) ( I V ) ~ } \left\{\begin{array}{l}
\text { Find }(\bar{x}, \bar{y}) \in K \times K \text { such that for each } i \in I, \\
\bar{x}_{i} \in B_{i}(\bar{x}), \bar{y}_{i} \in F_{i}(\bar{x}) \text { and } \\
\Phi_{i}\left(\bar{x}, \bar{y}, u_{i}\right) \not-\operatorname{int} C_{i}(\bar{x}, \bar{y}) \quad \text { for all } u_{i} \in A_{i}(\bar{x}) .
\end{array}\right.\right.
\end{gathered}
$$

It is clear that every solution of (SGVQEP)(I) (respectively, (SGVQEP)(III)) is a solution of (SGVQEP)(II) (respectively, (SGVQEP)(IV)) but converse assertion does not hold.

When $A_{i}(x)=B_{i}(x)$ for all $x \in K$ for each $i \in I$, then above problems are considered and studied in $[29,36]$ in the setting of topological vector spaces.

A problem similar to (SGVQEP)(I) and some other types of systems of generalized vector quasi-equilibrium problems are studied in [20] in the setting of locally $G$-convex uniform spaces.

If for each $i \in I$ and for all $x, y \in K, B_{i}(x)=A_{i}(x), F_{i}(x)=K_{i}, C_{i}(x, y)=$ $C_{i}(x)$ and $\Phi_{i}\left(x, y, u_{i}\right)$ is independent of the variable $y$, that is, $\Phi_{i}\left(x, y, u_{i}\right)$ is a multivalued map of two variables $x$ and $u_{i}$, then (SGVQEP)(IV) is introduced and studied by Ansari and Khan [4]. They established the existence results for a solution of such problem. As an application of their problem, they derived existence results for a solution of (Debreu VEP)(II) (see below) for nonconvex and nondifferentiable (in some sense) vector-valued functions. Furthermore, if for each $i \in I$ and for all $x \in K, F_{i}(x)=A_{i}(x)=B_{i}(x)=K$, then (SGVQEP)(IV) is considered and studied by Ansari et al. [6]. Some existence results for solutions of such problem are established in [6] with applications to Nash equilibrium problem [35] for vector-valued functions.

For each $i \in I$, if $\Phi_{i}$ is a single valued map, then (SGVQEP)(I) and (SGVQEP)(II), and (SGVQEP)(III) and (SGVQEP)(IV), respectively, reduce to the following systems of vector quasi-equilibrium problems (for short, (SVQEPs)).

$$
(\operatorname{SVQEP})(\mathrm{I})\left\{\begin{array}{l}
\text { Find }(\bar{x}, \bar{y}) \in K \times K \text { such that for each } i \in I, \\
\bar{x}_{i} \in B_{i}(\bar{x}), \bar{y}_{i} \in F_{i}(\bar{x}) \text { and } \\
\Phi_{i}\left(\bar{x}, \bar{y}, u_{i}\right) \in C_{i}(\bar{x}, \bar{y}) \quad \text { for all } u_{i} \in A_{i}(\bar{x})
\end{array}\right.
$$


$(\mathrm{SVQEP})(\mathrm{II}) \quad\left\{\begin{array}{l}\text { Find }(\bar{x}, \bar{y}) \in K \times K \text { such that for each } i \in I, \\ \bar{x}_{i} \in B_{i}(\bar{x}), \bar{y}_{i} \in F_{i}(\bar{x}) \text { and } \\ \Phi_{i}\left(\bar{x}, \bar{y}, u_{i}\right) \notin-\operatorname{int} C_{i}(\bar{x}, \bar{y}) \quad \text { for all } u_{i} \in A_{i}(\bar{x}) .\end{array}\right.$

Of course, $(\mathrm{SVQEP})(\mathrm{I})$ is more general than (SVQEP)(II) as every solution of (SVQEP)(I) is a solution of (SVQEP)(II) but converse assertion is not true.

For each $i \in I$, if $A_{i}(x)=B_{i}(x)$ for all $x \in K$ and $C_{i}(x, y)$ is independent of $y$, that is, $C_{i}(x, y)$ is a single variable multivalued map, then (SVQEP)(II) is considered and studied in [1]. Furthermore, if $F_{i}(x)=K$ for all $x \in K$, then (SVQEP)(II) is considered and studied in [2].

If for each $i \in I$ and for all $x, y \in K, B_{i}(x)=A_{i}(x), F_{i}(x)=K_{i}, C_{i}(x, y)=$ $C_{i}(x)$ and $\Phi_{i}\left(x, y, u_{i}\right)$ is independent of the variable $y$, that is, $\Phi_{i}\left(x, y, u_{i}\right)$ is a single valued map of two variables $x$ and $u_{i}$, then the existence of solutions of (SVQEP)(II) is studied in [3] with application to (Debreu VEP)(II).

Furthermore, if for each $i \in I$ and for all $x \in K, F_{i}(x)=A_{i}(x)=K_{i}$. Then $(\mathrm{SVQEP})(\mathrm{II})$ is studied in $[5,22]$.

For each $i \in I$, let $\varphi_{i}: K \rightarrow Y_{i}$ be a vector-valued map, $F_{i}(x)=K$ for all $x \in K$ and $\Phi_{i}\left(x, y, u_{i}\right)=\varphi_{i}\left(x^{i}, u_{i}\right)-\varphi_{i}(x)$ for all $\left(x, y, u_{i}\right) \in K \times K \times K_{i}$, then (SVQEP)(I) and (II) reduce to the following two classes of Debreu type equilibrium problems [11] for vector-valued maps.

(Debreu VEP)(I) $\left\{\begin{array}{l}\text { Find } \bar{x} \in K \text { such that for each } i \in I, \bar{x}_{i} \in B_{i}(\bar{x}) \text { and } \\ \varphi_{i}\left(\bar{x}^{i}, u_{i}\right)-\varphi_{i}(\bar{x}) \in C_{i}(\bar{x}) \quad \text { for all } u_{i} \in A_{i}(\bar{x}) .\end{array}\right.$

(Debreu VEP)(II) $\left\{\begin{array}{l}\text { Find } \bar{x} \in K \text { such that for each } i \in I, \bar{x}_{i} \in B_{i}(\bar{x}) \text { and } \\ \varphi_{i}\left(\bar{x}^{i}, u_{i}\right)-\varphi_{i}(\bar{x}) \notin-\operatorname{int} C_{i}(\bar{x}) \quad \text { for all } u_{i} \in A_{i}(\bar{x}) .\end{array}\right.$

From the above special cases, it is clear that our (SGVQEPs) are more general and unifying models of several problems studied in the literature.

Definition 5.1. Let $W$ be a topological semilattice space with path-connected intervals, $Z$ be a topological vector space and $M$ be a nonempty $\Delta$-convex subset of $W$. Let $P: M \times M \rightarrow 2^{Z}$ be a multivalued map such that for each $(x, y) \in M \times M$, $P(x, y)$ is a proper closed, convex cone. For all $(x, y) \in M \times M$, a multivalued map $\Psi: M \times M \times M \rightarrow 2^{Z} \backslash\{\emptyset\}$ is called

(a) $P(x, y)$ - $\Delta$-quasiconvex (in short, $P(x, y)-\Delta$-QC) if for any finite subset $N=$ $\left\{u_{1}, \ldots, u_{m}\right\} \subseteq M$ and for all $u \in \Delta(N)$, there exists $j \in\{1, \ldots, m\}$ such that $\Psi\left(x, y, u_{j}\right) \subseteq \Psi(x, y, u)+P(x, y)$;

(b) $P(x, y)$ - $\Delta$-quasiconvex-like (in short, $P(x, y)$ - $\Delta$-QCL) if for any finite subset $N=\left\{u_{1}, \ldots, u_{m}\right\} \subseteq M$ and for all $u \in \Delta(N)$, there exists $j \in\{1, \ldots, m\}$ such that $\Psi\left(x, y, u_{j}\right) \subseteq \Psi(x, y, u)-P(x, y)$; 
Rest of the paper, unless otherwise specified, for each $i \in I, Y_{i}$ is a topological vector space, $B_{i}: K \rightarrow 2^{K_{i}}$ is a multivalued map and $A_{i}: K \rightarrow 2^{K_{i}}$ is a lower semicontinuous multivalued map with nonempty values. For each $i \in I$, let $F_{i}: K \rightarrow 2^{K_{i}}$ be a multivalued map with nonempty $\Delta$-convex values such that for all $u_{i} \in K_{i}, F_{i}^{-1}\left(u_{i}\right)$ is compactly open in $K$.

We present the existence results for solutions of (SGVQEP) in the setting of topological semilattice spaces.

Theorem 5.1. For each $i \in I$, let $\Phi_{i}: K \times K \times K_{i} \rightarrow 2^{Y_{i}}$ be a lower semicontinuous multivalued map with nonempty values such that the following conditions are satisfied:

(i) For all $x \in K, \mathrm{CO}_{\Delta}\left(A_{i}(x)\right) \subseteq B_{i}(x)$.

(ii) For all $(x, y) \in K \times K, \Phi_{i}\left(x, y, x_{i}\right) \subseteq C_{i}(x, y)$, where $x_{i}$ is the ith component of $x$.

(iii) For all $(x, y) \in K \times K$, the set $\left\{u_{i} \in K_{i}: \Phi_{i}\left(x, y, u_{i}\right) \nsubseteq C_{i}(x, y)\right\}$ is $\Delta$-convex.

(iv) For each $i \in I, C_{i}: K \times K \rightarrow 2^{Y_{i}}$ is an upper semicontinuous multivalued map such that for all $(x, y) \in K \times K, C_{i}(x, y)$ is a proper closed convex cone.

(v) For all $M_{i}, N_{i} \in\left\langle K_{i}\right\rangle$, there exist compact $\Delta$-convex subsets $L_{M_{i}}, L_{N_{i}} \subseteq K_{i}$ containing $M_{i}$ and $N_{i}$, respectively, and nonempty compact subsets $\tilde{D}$ and $\hat{D}$ of $K$ such that for all $(x, y) \in K \times K \backslash \tilde{D} \times \hat{D}$ and for each $i \in I$, there exists $\left(\tilde{u}_{i}, \tilde{v}_{i}\right) \in L_{M_{i}} \times L_{N_{i}}$ satisfying $\tilde{u}_{i} \in A_{i}(x), \Phi_{i}\left(x, y, \tilde{u}_{i}\right) \nsubseteq C_{i}(x, y)$ and $\tilde{v}_{i} \in F_{i}(x)$.

Then $(S G V Q E P)(I)$ has a solution.

Proof. For each $i \in I$ and for all $(x, y) \in K \times K$, define a multivalued map $P_{i}: K \times K \rightarrow 2^{K_{i}}$ by

$$
P_{i}(x, y)=\left\{u_{i} \in K_{i}: \Phi_{i}\left(x, y, u_{i}\right) \nsubseteq C_{i}(x, y)\right\} .
$$

By condition (iii), for each $i \in I$ and for all $(x, y) \in K \times K, P_{i}(x, y)$ is $\Delta$ convex. Therefore, condition (ii) implies that $x_{i} \notin P_{i}(x, y)=\mathrm{CO}_{\Delta}\left(P_{i}(x, y)\right)$ for all $(x, y) \in K \times K$ and for each $i \in I$. Following the same argument as in the proof of Theorem 3.2.1 in [29], for each $i \in I, P_{i}$ has an open graph in $K \times K \times K_{i}$. Therefore, for each $i \in I$, the set $P_{i}^{-1}\left(u_{i}\right)=\left\{(x, y) \in K \times K: F_{i}\left(x, y, u_{i}\right) \nsubseteq\right.$ $\left.C_{i}(x, y)\right\}$ is open and hence compactly open in $K$ for all $u_{i} \in K_{i}$. Since for each $i \in I, A_{i}: K \rightarrow 2^{K_{i}}$ is lower semicontinuous and $P_{i}: K \times K \rightarrow 2^{K_{i}}$ has open graph, we have $P_{i} \cap A_{i}: K \times K \rightarrow 2^{K_{i}}$ is lower semicontinuous [[9], pp. 59-61]. 
Then it is easy to verify that the set $\left\{(x, y) \in K \times K: P_{i}(x, y) \cap A_{i}(x)=\emptyset\right\}$ is open and hence compactly open.

Condition (v) of Theorem 3.3 is followed from condition (v). Hence by Theorem 3.3, there exists $(\bar{x}, \bar{x}) \in K \times K$ such that for each $i \in I, \bar{x}_{i} \in B_{i}(\bar{x}), \bar{y}_{i} \in F_{i}(\bar{x})$ and $P_{i}(x, y) \cap A_{i}(x)=\emptyset$, that is, $\Phi_{i}\left(\bar{x}, \bar{y}, u_{i}\right) \subseteq C_{i}(\bar{x}, \bar{y})$ for all $u_{i} \in A_{i}(\bar{x})$.

Theorem 5.2. For each $i \in I$, let $X_{i}$ be a metrizable topological semilattice with path-connected intervals. For each $i \in I$, let $\Phi_{i}: K \times K \times K_{i} \rightarrow 2^{Y_{i}}$ be a upper semicontinuous multivalued map with nonempty compact values such that the following conditions are satisfied:

(i) For all $x \in K, \mathrm{CO}_{\Delta}\left(A_{i}(x)\right) \subseteq B_{i}(x)$.

(ii) For all $(x, y) \in K \times K, \Phi_{i}\left(x, y, x_{i}\right) \cap C_{i}(x, y) \neq \emptyset$, where $x_{i}$ is the ith component of $x$.

(iii) For all $(x, y) \in K \times K$, the set $\left\{u_{i} \in K_{i}: \Phi_{i}\left(x, y, u_{i}\right) \cap C_{i}(x, y)=\emptyset\right\}$ is $\Delta$-convex.

(iv) For each $i \in I, C_{i}: K \times K \rightarrow 2^{Y_{i}}$ is an upper semicontinuous multivalued map such that for all $(x, y) \in K \times K, C_{i}(x, y)$ is a proper, closed convex cone.

(v) For all $M_{i}, N_{i} \in\left\langle K_{i}\right\rangle$, there exist compact $\Delta$-convex subsets $L_{M_{i}}, L_{N_{i}} \subseteq K_{i}$ containing $M_{i}$ and $N_{i}$, respectively, and nonempty compact subsets $\tilde{D}$ and $\hat{D}$ of $K$ such that for all $(x, y) \in K \times K \backslash \tilde{D} \times \hat{D}$ and for each $i \in I$, there exists $\left(\tilde{u}_{i}, \tilde{v}_{i}\right) \in L_{M_{i}} \times L_{N_{i}}$ satisfying $\tilde{u}_{i} \in A_{i}(x), \Phi_{i}\left(x, y, \tilde{u}_{i}\right) \cap C_{i}(x, y)=\emptyset$ and $\tilde{v}_{i} \in F_{i}(x)$.

Then (SGVQEP)(II) has a solution.

Proof. For each $i \in I$ and for all $(x, y) \in K \times K$, define a multivalued map $P_{i}: K \times K \rightarrow 2^{K_{i}}$ by

$$
P_{i}(x, y)=\left\{y_{i} \in K_{i}: \Phi_{i}\left(x, y, u_{i}\right) \cap C_{i}(x, y)=\emptyset\right\} .
$$

Since each $\Phi_{i}$ is upper semicontinuous with nonempty compact values and each $C_{i}$ is also upper semicontinuous, by using the same argument as in [8], we obtain that the graph of $P_{i}$ is open. Then by following the same argument as in the proof of Theorem 5.1, we get the conclusion.

Theorem 5.3. For each $i \in I$, let $C_{i}: K \times K \rightarrow 2^{Y_{i}}$ be a multivalued map such that for all $(x, y) \in K \times K, C_{i}(x, y)$ is a proper, closed convex cone with nonempty interior. For each $i \in I$, let $\Phi_{i}: K \times K \times K_{i} \rightarrow 2^{Y_{i}}$ be a lower semicontinuous multivalued map with nonempty values such that the following conditions are satisfied:

(i) For all $x \in K, \mathrm{CO}_{\Delta}\left(A_{i}(x)\right) \subseteq B_{i}(x)$. 
(ii) For all $(x, y) \in K \times K, \Phi_{i}\left(x, y, x_{i}\right) \cap\left(-\operatorname{int} C_{i}(x, y)\right)=\emptyset$, where $x_{i}$ is the ith component of $x$.

(iii) For all $(x, y) \in K \times K$, the set $\left\{u_{i} \in K_{i}: \Phi_{i}\left(x, y, u_{i}\right) \cap\left(-\operatorname{int} C_{i}(x, y)\right) \neq \emptyset\right\}$ is $\Delta$-convex.

(iv) For each $i \in I, W_{i}: K \times K \rightarrow 2^{Y_{i}}$ is an upper semicontinuous multivalued map defined as $W_{i}(x, y)=Y_{i} \backslash\left(-\operatorname{int} C_{i}(x, y)\right)$ for all $(x, y) \in K \times K$.

(v) For all $M_{i}, N_{i} \in\left\langle K_{i}\right\rangle$, there exist compact $\Delta$-convex subsets $L_{M_{i}}, L_{N_{i}} \subseteq K_{i}$ containing $M_{i}$ and $N_{i}$, respectively, and nonempty compact subsets $\tilde{D}$ and $\hat{D}$ of $K$ such that for all $(x, y) \in K \times K \backslash \tilde{D} \times \hat{D}$ and for each $i \in I$, there exists $\left(\tilde{u}_{i}, \tilde{v}_{i}\right) \in L_{M_{i}} \times L_{N_{i}}$ satisfying $\tilde{u}_{i} \in A_{i}(x), \Phi_{i}\left(x, y, \tilde{u}_{i}\right) \cap\left(-\operatorname{int} C_{i}(x, y)\right) \neq \emptyset$ and $\tilde{v}_{i} \in F_{i}(x)$.

Then (SGVQEP)(III) has a solution.

Proof. For each $i \in I$ and for all $(x, y) \in K \times K$, define a multivalued map $P_{i}: K \times K \rightarrow 2^{K_{i}}$ by

$$
P_{i}(x, y)=\left\{u_{i} \in K_{i}: \Phi_{i}\left(x, y, u_{i}\right) \cap\left(-\operatorname{int} C_{i}(x, y)\right) \neq \emptyset\right\} .
$$

Since each $\Phi_{i}$ is lower semicontinuous and $W_{i}$ is upper semicontinuous, by using the argument same as in the proof of Theorem 5.2, that the graph of $P_{i}$ is open. Then by following the same argument as in the proof of Theorem 5.1, we get the conclusion.

Theorem 5.4. For each $i \in I$, let $X_{i}$ be a metrizable topological semilattice with path-connected intervals and $C_{i}: K \times K \rightarrow 2^{Y_{i}}$ be a multivalued map such that for all $(x, y) \in K \times K, C_{i}(x, y)$ is a proper, closed convex cone with nonempty interior. . For each $i \in I$, let $\Phi_{i}: K \times K \times K_{i} \rightarrow 2^{Y_{i}}$ be a upper semicontinuous multivalued map with nonempty compact values such that the following conditions are satisfied:

(i) For all $x \in K, \mathrm{CO}_{\Delta}\left(A_{i}(x)\right) \subseteq B_{i}(x)$.

(ii) For all $(x, y) \in K \times K, \Phi_{i}\left(x, y, x_{i}\right) \nsubseteq-\operatorname{int} C_{i}(x, y)$, where $x_{i}$ is the ith component of $x$.

(iii) For all $(x, y) \in K \times K$, the set $\left\{u_{i} \in K_{i}: \Phi_{i}\left(x, y, u_{i}\right) \subseteq-\operatorname{int} C_{i}(x, y)\right\}$ is $\Delta$-convex.

(iv) For each $i \in I, W_{i}: K \times K \rightarrow 2^{Y_{i}}$ is an upper semicontinuous multivalued map defined as $W_{i}(x, y)=Y_{i} \backslash\left(-\operatorname{int} C_{i}(x, y)\right)$ for all $(x, y) \in K \times K$.

(v) For all $M_{i}, N_{i} \in\left\langle K_{i}\right\rangle$, there exist compact $\Delta$-convex subsets $L_{M_{i}}, L_{N_{i}} \subseteq K_{i}$ containing $M_{i}$ and $N_{i}$, respectively, and nonempty compact subsets $\tilde{D}$ and $\hat{D}$ of $K$ such that for all $(x, y) \in K \times K \backslash \tilde{D} \times \hat{D}$ and for each $i \in I$, there exists $\left(\tilde{u}_{i}, \tilde{v}_{i}\right) \in L_{M_{i}} \times L_{M_{i}}$ satisfying $\tilde{u}_{i} \in A_{i}(x), \Phi_{i}\left(x, y, \tilde{u}_{i}\right) \subseteq-\operatorname{int} C_{i}(x, y)$ and $\tilde{v}_{i} \in F_{i}(x)$. 
Then (SGVQEP)(IV) has a solution.

Proof. For each $i \in I$ and for all $(x, y) \in K \times K$, define a multivalued map $P_{i}: K \times K \rightarrow 2^{K_{i}}$ by

$$
P_{i}(x, y)=\left\{u_{i} \in K_{i}: \Phi_{i}\left(x, y, u_{i}\right) \subseteq-\operatorname{int} C_{i}(x, y)\right\} .
$$

Following the same argument as in the proof of Theorems 5.1-5.3, we get the conclusion.

\section{Remark 5.1.}

(a) If for each $i \in I$ and for all $(x, y) \in K \times K, \Phi_{i}$ is $C_{i}(x, y)$ - $\Delta$-QC, then the set $\mathcal{K}_{i}=\left\{u_{i} \in K_{i}: \Phi_{i}\left(x, y, u_{i}\right) \nsubseteq C_{i}(x, y)\right\}$ is $\Delta$-convex, that is, condition (iii) of Theorem 5.1 is satisfied.

Indeed, let $N_{i}=\left\{u_{i_{1}}, \ldots, u_{i_{m}}\right\}$ be any finite subset of $\mathcal{K}_{i}$. Then we show that $\Delta\left(N_{i}\right) \subseteq \mathcal{K}_{i}$ which implies that $\mathcal{K}_{i}$ is $\Delta$-convex. Suppose contrary that $\Delta\left(N_{i}\right) \nsubseteq \mathcal{K}_{i}$. Then for some $\tilde{u}_{i} \in \Delta\left(N_{i}\right), \tilde{u}_{i} \notin \mathcal{K}_{i}$, that is, $\Phi_{i}\left(x, y, \tilde{u}_{i}\right) \subseteq$ $C_{i}(x, y)$. Since each $\Phi_{i}$ is $C_{i}(x, y)-\Delta$-QC, for all $z_{i} \in \Delta\left(N_{i}\right)$, there exists $j \in\{1, \ldots, m\}$ such that

$$
\Phi_{i}\left(x, y, u_{i_{j}}\right) \subseteq \Phi_{i}\left(x, y, z_{i}\right)+C_{i}(x, y)
$$

and so

$$
\Phi_{i}\left(x, y, u_{i_{j}}\right) \subseteq \Phi_{i}\left(x, y, \tilde{u}_{i}\right)+C_{i}(x, y) \subseteq C_{i}(x, y)+C_{i}(x, y) \subseteq C_{i}(x, y) .
$$

Therefore, $u_{i_{j}} \notin \mathcal{K}_{i}$ for $j \in\{1, \ldots, m\}$ which contradicts to our assumption that each $u_{i_{j}} \in \mathcal{K}_{i}$ for all $j \in\{1, \ldots, m\}$. Hence $\mathcal{K}_{i}$ is $\Delta$-convex.

(b) If for each $i \in I$ and for all $(x, y) \in K \times K, \Phi_{i}$ is $C_{i}(x, y)-\Delta$-QCL, then the set $\mathcal{K}_{i}=\left\{u_{i} \in K_{i}: \Phi_{i}\left(x, y, u_{i}\right) \cap C_{i}(x, y)=\emptyset\right\}$ is $\Delta$-convex, that is, condition (iii) of Theorem 5.2 is satisfied.

Indeed, let $N_{i}=\left\{u_{i_{1}}, \ldots, u_{i_{m}}\right\}$ be any finite subset of $\mathcal{K}_{i}$. Then we show that $\Delta\left(N_{i}\right) \subseteq \mathcal{K}_{i}$ which implies that $\mathcal{K}_{i}$ is $\Delta$-convex. Since $u_{i_{j}} \in \mathcal{K}_{i}$ for all $j=1, \ldots, m$, we have $\Phi_{i}\left(x, y, u_{i_{j}}\right) \cap C_{i}(x, y)=\emptyset$. Since each $\Phi_{i}$ is $C_{i}(x, y)-\Delta$-QCL, for any $\tilde{u}_{i} \in \Delta\left(N_{i}\right)$, there exists $j \in\{1, \ldots, m\}$ such that

$$
\Phi_{i}\left(x, y, \tilde{u}_{i}\right) \subseteq \Phi_{i}\left(x, y, u_{i_{j}}\right)-C_{i}(x, y)
$$

and so

$$
\Phi_{i}\left(x, y, \tilde{u}_{i}\right) \cap C_{i}(x, y) \subseteq\left(\Phi_{i}\left(x, y, u_{i_{j}}\right)-C_{i}(x, y)\right) \cap C_{i}(x, y)=\emptyset .
$$

Therefore, $\Phi_{i}\left(x, y, \tilde{u}_{i}\right) \cap C_{i}(x, x)=\emptyset$ and thus $\tilde{u}_{i} \in \mathcal{K}_{i}$. Hence $\mathcal{K}_{i}$ is $\Delta$ convex. 
(c) By using the same argument as in (a) and (b), we can easily show that for each $i \in I$ and for all $(x, y) \in K \times K$, the set $\left\{u_{i} \in K_{i}: \Phi_{i}\left(x, y, u_{i}\right) \cap\right.$ $\left.\left(-\operatorname{int} C_{i}(x, y)\right)=\emptyset\right\}$ and $\left\{u_{i} \in K_{i}: \Phi_{i}\left(x, y, u_{i}\right) \subseteq-\right.$ int $\left.C_{i}(x, y)\right\}$ are $\Delta$-convex, that is, condition (iii) of Theorems 5.3 and 5.4 is satisfied if $\Phi_{i}$ is $C_{i}(x, y)-\Delta$-QC and $C_{i}(x, y)-\Delta$-QCL, respectively.

Remark 5.2. If for each $i \in I, \Phi_{i}: K \times K \times K_{i} \rightarrow Y_{i}$ is a single-valued map, then from Theorems 5.1-5.4 we can easily derive the existence results for solutions of (SVQEP) considered in previous section.

Remark 5.3. Theorems 5.1-5.4 extend Theorems 3.2.1-3.2.4 in [29] and Theorems 3.1-3.4 with Remarks 3.2 and 3.5 in [36] to topological semilattice spaces.

\section{REFERENCES}

1. Q. H. Ansari, Existence of solutions of systems of generalized implicit vector quasiequilibrium problems, J. Math. Anal. Appl., 341 (2008), 1271-1283.

2. S. Al-Homidan, Q. H. Ansari and S. Schaible, Existence of solutions of systems of generalized implicit vector variational inequalities, J. Optim. Theory Appl., 134 (2007), 515-531.

3. Q. H. Ansari, W. K. Chan and X. Q. Yang, The system of vector quasi-equilibrium problems with applications, J. Global Optim., 29(1) (2004), 45-57.

4. Q. H. Ansari and Z. Khan, System of generalized vector quasi-equilibrium problems with applications, in Mathematical Analysis and Applications, Edited by S. Nanda and G. P. Rajasekhar, Narosa Publication House, New Delhi, pp. 1-13, 2004.

5. Q. H. Ansari, S. Schaible and J. C. Yao, The system of vector equilibrium problems and its applications, J. Optim. Theory Appl., 107(3) (2000), 547-557.

6. Q. H. Ansari, S. Schaible and J. C. Yao, The system of generalized vector equilibrium problems with applications, J. Global Optim., 22 (2002), 3-16.

7. Q. H. Ansari and J. C. Yao, A fixed point theorem and its applications to a system of variational inequalities, Bull. Austral. Math. Soc., 59 (1999), 433-442.

8. Q. H. Ansari and J. C. Yao, An existence result for the generalized vector equilibrium problem, Appl. Math. Lett., 12 (1999), 53-56.

9. K. C. Border, Fixed Point Theorems with Applications to Economics and Game Theory, Cambridge University Press, Cambridge, 1985.

10. S. S. Chang, B. S. Lee, X. Wu, Y. J. Chao and G. M. Lee, On the generalized quasi-variational inequality problems, J. Math. Anal. Appl., 203 (1996), 686-711.

11. G. Debreu, A social equilibrium existence theorem, Proc. Nat. Acad. Sci. USA, 38 (1952), 886-893. 
12. X. P. Ding, New H-KKM theorems and their applications to geometric property, coincidence theorems, minimax inequalitiy and maximal elements, Indian J. Pure Appl. Math., 26(1) (1995), 1-19.

13. X. P. Ding, Coincidence theorems in topological spaces and their applications, Appl. Math. Lett., 12(7) (1999), 99-105.

14. X. P. Ding, Existence of solutions for quasi-equilibrium problems in noncompact topological spaces, Comput. Math. Appl., 39 (2000), 13-21.

15. X. P. Ding, Continuous section, collectively fixed points and system of coincidence theorems in product topological spaces, Acta Math. Sinica, English Series, 22 (2006), 1629-1638.

16. X. P. Ding, W. K. Kim and K. K. Tan, A selection theorem and its applications, Bull. Austral. Math. Soc., 46 (1992), 205-212.

17. X. P. Ding, J. Y. Park and I. H. Jung, Fixed point theorems on product topological spaces and applications, Positivity, 8 (2004), 315-326.

18. X. P. Ding and K. K. Tan, On equilibria of noncompact generalized games, J. Math. Anal. Appl., 177 (1993), 226-238.

19. X. P. Ding and J. C. Yao, Maximal element theorems with applications to generalized games and systems of generalized vector quasi-equilibrium problems in $G$-convex spaces, J. Optim. Theory Appl., 126(3) (2005), 571-588.

20. X. P. Ding and J. C. Yao and L. J. Lin, Solitions of system of generalized vector quasiequilibrium problems in locally $G$-convex uniform spaces, J. Math. Anal. Appl., 298 (2004), 398-410.

21. K. Fan, Some properties of convex sets related fixed point theorems, Math. Ann., 266 (1984), 519-537.

22. Y. P. Fang, N.-J. Hunag and J. K. Kim, Existence results for systems of vector equilibrium problems, J. Global Optim., 35 (2006), 71-83.

23. C. D. Horvath and J. V. L. Ciscar, Maximal elements and fixed points for binary relations on topological ordered spaces, J. Math. Econom., 25 (1996), 291-306.

24. W. K. Kim and K. K. Tan, New existence theorems of equilibria and applications, Nonlinear Anal., 47 (2001), 531-542.

25. K. Q. Lan and J. Webb, New fixed point theorems for a family of mappings and applications to problems on sets with convex sections, Proc. Amer. Math. Soc., 126 (1998), 1127-1132.

26. L.-J. Lin, Mathematical programming with system of equilibrium constraints, $J$. Global Optim., 37 (2007), 275-286.

27. L.-J. Lin, System of generalized vector quasi-equilibrium problems with applications to fixed point theorems for a family of nonexpansive multivalued mappings, J. Global Optim., 34 (2006), 15-32. 
28. L.-J. Lin, and Q. H. Ansari, Collective fixed points and maximal elements with applications to abstract economies, J. Math. Anal. Appl., 296 (2004), 455-472.

29. L.-J. Lin, L.-F. Chen and Q. H. Ansari, Generalized abstract economy and systems of generalized vector quasi-equilibrium problems, J. Computat. Appl. Math., 208 (2007), 341-353.

30. L.-J. Lin and Y.-H. Liu, The study of abstract economics with two constraints correspondences, J. Optim. Theory Appl., 137 (2008), 41-52.

31. L.-J. Lin, S. Park and Z.-T. Yu, Remarks on fixed points, maximal elements, and equilibria of generalized games, J. Math. Anal. Appl., 233 (1999), 581-596.

32. L.-J. Lin, Z.-T. Yu, Q. H. Ansari and L.-P. Lai, Fixed point and maximal element theorems with applications to abstract economies and minimax inequalities, J. Math. Anal. Appl., 284 (2003), 656-671.

33. Z. Lin and J. Yu, The existence of solutions for the system of generalized vector quasi-equilibrium problems, Appl. Math. Lett., 18 (2005), 415-422.

34. Q. Luo, Ky Fan's section theorem and its applications in topological ordered spaces, Appl. Math. Lett., 17 (2004), 1113-1119.

35. J. Nash, Non-cooperative games, Ann. Math., 54 (1951), 286-295.

36. J.-W. Peng, H.-W. J. Lee and X.-M. Yang, On system of generalized vector quasiequilibrium problems with set-valued maps, J. Global Optim., 36 (2006), 139-158.

37. W. Shafer and H. Sonnenschein, Equilibrium in abstract economics without ordered preference, J. Math. Econom., 2 (1975), 345-348.

38. M. H. Shih and K. K. Tan, Non-compact sets with convex sections, Pacific J. Math., 119 (1985), 473-479.

39. S. P. Singh, E. Tarafdar and B. Watson, A generalized fixed point theorem and equilibrium point of an abstract economy, J. Computat. Appl. Math., 113 (2000), 65-71.

40. E. Tarafdar, On nonlinear variational inequalities, Proc. Amer. Math. Soc., 67 (1977), 95-98.

41. E. Tarafdar, A fixed point theorem ans equilibrium point of an abstract economy, $J$. Math. Econom., 20 (1991), 211-218.

42. A. Tychonoff, Ein Fixpunktsatz, Math. Ann., 111 (1935), 767-776.

43. X. Wu and S. Shen, A further generalization of Yannelis-Prabhakar's continuous selection theorem and its applications, J. Math. Anal. Appl., 196 (1996), 61-74.

44. N. C. Yannelis and N. D. Prabhakar, Existence of maximal elements and equilibria in linear topological spaces, J. Math. Econom., 12 (1983), 233-245. 
Suliman Al-Homidan

Department of Mathematics \& Statistics,

King Fahd University of Petroleum \& Minerals,

P. O. Box 119, Dhahran,

Saudi Arabia

E-mail: homidan@kfupm.edu.sa

Qamrul Hasan Ansari

Department of Mathematics,

Aligarh Muslim University,

Aligarh 202002 ,

India

E-mail: qhansari@gmail.com 